system in vitro was correlated with origin of synoviocytes. Moreover the relation between invasive growth of FLS in the transwell system and growth characteristics and expression of MMP $1-14,17,19$, cathepsin K, TIMP-1 and -2 was determined.

Methods FLS were derived from 56 patients (30 rheumatoid arthritis (RA), 17 osteoarthritis (OA), 9 avascular necrosis (AVN) or fractures). Invasive growth of FLS through a collagen matrix was measured in a transwell system coated with matrigel. The number of cells grown through the matrix and the transwell membrane were counted. Growth rate was determined by counting of FLS after seven days of culturing. Expression of MMP's, cathepsin-K and TIMP's was investigated using RT-PCR and related to expression of a household gene, beta-actin.

Results FLS from RA invaded more easily in vitro than FLS from OA and AVN (median: RA: 4788 (cells grown through matrix and transwell membrane), OA: 1875; $\mathrm{p}<0.001$ and AVN: $1530 ; p=0.014$ respectively). The median rate of proliferation of RA FLS was 0.27 per day compared to OA 0.22 per day $(\mathrm{p}=0.012)$ and AVN and fractures 0.25 per day (RA versus AVN: $p=0.242$; OA versus AVN: $p=0.553$ ), but there was no correlation between rate of proliferation and invasive growth in vitro. FLS that expressed MMP-1, MMP-3 or MMP-10 were significantly more invasive (median number of invasive cells: 3835 , 4248, 4990, respectively) than cells that did not express MMP1, MMP-3 or MMP-10 (1605, $\mathrm{p}=0.03 ; 1970, \mathrm{p}=0.004$; $2360, p=0.012$, respectively). Expression of the other MMP's, cathepsin-K and TIMP's did not show a significant relationship with invasive growth. Expression of MMP-9 showed a trend with higher expression in more invasive cells $(\mathrm{p}=0.066)$. There was also a significant relationship between the expression of MMP-1 and MMP-9 and a diagnosis of RA (both $p=0.013$ ).

Conclusion FLS of RA invade more easily in a matrigel matrix than OA FLS. This is not due to a greater rate of proliferation of RA FLS than OA FLS, although FLS from RA patients have a $25 \%$ higher rate of proliferation than FLS from OA patients. A significant relationship exists between the expression of MMP-1, MMP-3 and MMP-10 and invisive growth in a matrigel transwell system and this relationship was nearly significant for MMP-9. FLS of RA patients expressed significantly more often MMP-1 and MMP-9 than FLS from OA patients.

\section{OP0025 GLUCOSAMINE SULFATE INHIBITS NITRIC OXIDE AND STROMELYSIN PRODUCTION IN CARTILAGE CULTURES AND REVERSES IL-1 INHIBITION OF OSTEOARTHRITIC ARTICULAR CARTILAGE SYNTHESIS}

I Yaron, I Shirazi, R Judovich, M Yaron. Department of Rheumatology, Ichilov Hospital, Tel Aviv, Israel

10.1136/annrheumdis-2001.917

Background Glucosamine sulfate is used for the management of osteoarthritis of the knee.

The mechanism of its effects needs clarification.

Objectives To evaluate the effects of glucosamine sulfate on the production of nitric oxide (NO) and stromelysin (MMP3) and cartilage synthesis in human osteoarthritic cartilage in the presence of IL-1b or LPS.

Methods Cartilage was obtained in the operation theatre from patients undergoing total knee replacement for osteoarthritis, cut into small pieces and cultured in the presence or absence of IL$1 \mathrm{~b}$ or LPS.
NO in culture media was measured with the Griess reagent, MMP- 3 by ELISA and cartilage synthesis by ${ }^{35} \mathrm{~S}$ incorporation. Results IL-1b $(1 \mathrm{ng} / \mathrm{ml})$ and LPS $(3 \mu \mathrm{g} / \mathrm{ml})$ stimulated NO release in human osteoarthritic cartilage cultures about four fold. Glucosamine sulfate $(1 \mu \mathrm{g} / \mathrm{ml}, 10 \mu \mathrm{g} / \mathrm{ml}, 100 \mu \mathrm{g} / \mathrm{ml})$ inhibited stimulated NO production in a dose dependent manner, reaching statistical significance $(\mathrm{p}<0.05)$ at $100 \mu \mathrm{g} / \mathrm{ml}$ glucosamine sulfate $(60 \%$ and $38 \%$ inhibition respectively).

Glucosamine sulfate $(100 \mu \mathrm{g} / \mathrm{ml})$ significantly $(\mathrm{p}<0.05)$ inhibited MMP-3 release in IL-1b and LPS stimulated cartilage cultures. IL- $1 \mathrm{~b}$ and LPS inhibited cartilage synthesis by $70 \%$ and $50 \%$ respectively $(\mathrm{p}<0.001)$. Glucosamine sulfate reversed this inhibition in a dose dependent manner reaching statistical significance $(\mathrm{p}<0.05)$ at $10 \mu \mathrm{g} / \mathrm{ml}$ glucosamine sulfate.

Conclusion Glucosamine sulfate may protect human osteoarthritic articular cartilage by inhibiting local NO and MMP-3 production and by reversing IL-1b inhibition of cartilage synthesis.

\section{OP0026 IN VIVO INHIBITION OF ENDOGENOUS TGF-BETA ACTIVITY REDUCES OSTEOPHYTE SIZE AND CARTILAGE PROTEOGLYCAN CONTENT}

A Scharstuhl, P Van der Kraan, E Vitters, H Van Beuningen, W Van der Berg. Department of Rheumatology, University Medical Centre Nijmegen, Nijmegen, The Netherlands

\subsection{6/annrheumdis-2001.918}

Background Transforming Growth Factor-beta (TGF-beta) is considered to be a beneficial factor during cartilage repair. However, TGF-beta can also induce fibrosis and osteophytes. TGFbeta signalling starts with the binding of TGF-beta to the type II receptor, a constitutively active serine/threonine kinase. This complex then binds the type I receptor, which is subsequently phosphorylated by the type II receptor. This results in phosphorylation of downstream mediators by the type I receptor. Since the binding of TGF-beta to the extracellular domain of the type II receptor is one of high affinity, the soluble TGF-beta type II receptor, lacking cytoplasmic and membrane domains, was used as a TGF-beta antagonist.

Objectives The objective of this study was to determine the effect of inhibition of endogenous TGF-beta during an experimental model of cartilage damage.

Methods For the production of recombinant solRII, the methylotropic yeast Pichia pastoris was used. Intra-articular injection of papaine was used as a model for experimental cartilage damage and osteophyte formation. The effect of systemic delivery of solRII on endogenous TGF-beta was investigated in this model. Therefore, Alzet osmotic pumps containing either $10 \mathrm{mg}$ of solRII or pumps containing solvent were implanted i.p. in $\mathrm{C} 57 \mathrm{Bl} / 6$ mice. One day later, a $1.2 \%$ papaine solution was injected in the right knee joint. Knees were dissected on day 7 and processed for routine histology.

Results Injection of papaine into murine knee joints resulted in the formation of osteophytes and a decrease in proteoglycan (PG) content of non-calcified articular cartilage. Blocking of endogenous TGF-beta via systemic administration of solRII resulted in a reduction of $75 \%$ in osteophyte size compared to controls. Moreover, cartilage PG content was significantly decreased in solRII treated animals compared to control animals. Conclusion This study shows for the first time that inhibition of endogenous TGF-beta leads to a reduction in osteophyte size in the papaine model of cartilage damage and osteophyte formation. This finding implicates a direct role for TGF-beta in the 
formation of osteophytes, a major characteristic of osteoarthritis. Furthermore, an important role for TGF-beta in the regulation of cartilage PG content is suggested, because systemic solRII treatment resulted in a decreased PG content in the papaine model. In conclusion, TGF-beta seems essential in osteophyte formation and protects against cartilage damage.

\section{OP0027 MECHANICAL STRESS WITH SILICON ROD ALTERED CARTILAGINOUS DIFFERENTIATIATION IN PSEUDO- TENDON SHEATH}

Y Yutani, Y Yamano. Department of Orthopaedic Surgery, Osaka City University Medical School, Osaka, Japan

\subsection{6/annrheumdis-2001.919}

Background In a joint-like sliding surface, undifferentiated mesenchymal cells have been reported to show expression of cartilage-specific genes and cartilage-specific matrix production.

Objectives In flexor tendon injury, tendon grafting is performed. When primary repair is impossible, a tendon sheath is constructed using a silicon rod. This area forms a sliding surface between the rod and the surrounding tissue, and cartilaginous differentiation is expected.

Methods The subjects were 22- and 46-year-old male patients. The mean interval between the injuries and insertion of the silicon rod was 13 months, and the mean interval until the second operation after insertion of the silicon rod was 4 months. Tissue was obtained from the inner and outer layer of the newly formed tendon sheath tissue. Total RNA was extracted directly from each tissue. For quantitative RT-PCR, the non-radioisotope method was used.

Results Although aggrecan was absent at the outer layer, the ratio of the expression of this gene to the expression of GAPDH was 0.15 at the inner layer of the newly formed tendon sheath. Gene expression of type II collagen was seen at a ratio of 0.28 at the inner layer, although it was also absent at the outer layer. On examining the gene expression of type I collagen and TGF-? A were 0.49 and 0.17 , respectively. CD44 and HAS-2 gene expression were also confirmed.

Conclusion In the tissue evaluated in this study, the original tendon sheath had been destroyed, and new tissue formation was observed around the site of artificial tendon insertion. Generally, tissues in this region were considered just connective tissues. However, the newly synthsized tendon sheath showed expression of genes for aggrecan and type II collagen that were not observed in the surrounding tissue. These findings together with expression of TGF-?A suggest alterations in the tissue differentiation function in this area. In the tissue of this area, mechanical stimulation may have played an important role in the differentiation of mesenchymal tissue.

\section{OP0028 UP-REGULATED EXPRESSION OF THE DISINTEGRIN METALLOPROTEINASE MDC15 IN HUMAN OSTEOARTHRITIC CARTILAGE}

${ }^{1} \mathrm{~B}$ Böhm, ${ }^{2} \mathrm{~T}$ Aigner, ${ }^{3} \mathrm{CP}$ Blobel, ${ }^{1} \mathrm{JR}$ Kalden, ${ }^{1} \mathrm{H}$ Burkhardt. ${ }^{1}$ Internal Medicine III, Clinical Immunology and Rheumatology, Erlangen; ${ }^{2}$ Institute of Pathology, Cartilage Research Group, Erlangen, Germany; ${ }^{3}$ Memorial Sloan Kettering Cancer Institute, New York, USA

10.1136/annrheumdis-2001.920

Background MDC15 (or ADAM15) is a member of the family of membrane-bound disintegrin-metalloproteinases (a disintegrin and metalloproteinase: ADAM or MDC). ${ }^{1}$ Some ADAMs are involved in (patho-) physiological important processes such as remodelling of the extracellular matrix (e.g. proteolytic activity of the "aggrecanases") ${ }^{2}$ and the shedding of membrane-bound cytokines (e.g. TNF-alpha cleavage by TACE). ${ }^{3}$ By in situ hybridization we could show that MDC15 is markedly up-regulated in osteoarthritic (OA) cartilage. ${ }^{4}$ However, mRNA expression in normal articular cartilage was below detection level, suggesting an involvement of MDC15 in catabolic processes of the cartilage matrix.

Objectives In the present study we examined the expression of MDC15 on protein level in OA cartilage compared to normal articular cartilage.

Methods The expression of MDC15 on protein level was analysed on 33 OA cartilage specimens and 14 normal tissue samples using immunohistochemistry. For this purpose a polyclonal antiserum was generated in rabbits by immunisation with a synthetic cyclized peptide (15-mer) derived from the sequence containing the RGD-motive of the disintegrin domain of MDC15. The specificity of the affinity-purified antibody was tested using Western Blot analysis on COS-7 cell lysates that were transfected with a full-length MDC15 cDNA. Immunostaining revealed a single protein band of around $100 \mathrm{kD}$ in the transfected but not in the non-transfected COS-7 cells.

Results In OA cartilage MDC15 was strongly expressed in all samples analysed. MDC15 expression did not exhibit any preference for certain zones in OA cartilage. Analogous to the in situ hybridization studies, the protein could be detected already in the early stages of OA (Mankin grades 2-7). Less damaged cartilage of peripheral non-weight bearing areas also displayed strong signals well above those observed in normal aged cartilage. Strong expression of MDC15 was also present in tissues that were severely damaged (Mankin grades $>7$ ). In addition, MDC15 was found in secondary formed cartilage of osteophytes as well as metaplastic cartilage in the opened bone marrow (areas of complete cartilage erosion). In contrary, normal cartilage exhibited no or very low expression of MDC15.

Conclusion In this work we demonstrate that MDC15 expression is upregulated in OA cartilage not only on mRNA level but also on protein level. Human MDC15 is the only ADAM that contains an RGD sequence in its disintegrin domain by which it can interact with integrins ${ }^{5}$ thus interfering with cellcell or cell-matrix interactions. Both its proteolytic potential as well as integrin-binding properties suggest that MDC15 plays an important role in cartilage remodelling in pathophysiological processes. Taken together, the proposed functions of MDC15 and its upregulation in $\mathrm{OA}$, make this interesting multidomain molecule a potential candidate as a new therapeutic target.

\section{REFERENCES}

1 Kraetschmar J, Lum L, Blobel CP. J Biol Chem. 1996;271:4593

2 Tortorella MD, Burn TC, Pratta MA, Abbaszade I, Hollis JM, et al. Science 1999:284:1664

3 Moss ML, Jin SL, Milla ME, Bickett DM, Burkhart W, Carter HL, et al. Nature 1997:385:733

4 Boehm B, Aigner T, Gehrsitz A, Blobel CP, Kalden JR, Burkhardt H. Arthritis Rheum. 1999;42:1946

5 Nath D, Slocombe PM, Stephens PE, Warn A, Hutchinson GR, Yamada KM, et al. J Cell Sci. 1999;112:579 